\title{
CONSTRAINTS FOR CATACLYSMIC BINARY EVOLUTION AS DERIVED FROM SPACE DISTRIBUTIONS*
}

\author{
H. W. DUERBECK \\ Observatorium Hoher List der Universitäts-Sternwarte Bonn, Daun, F.R.G.
}

(Received 30 August, 1983)

\begin{abstract}
Space densities and galactic $z$-distributions of novae, recurrent novae, dwarf novae and symbiotic stars are newly determined and discussed in the context of earlier determinations. The data are then compared with the distributions of single and binary stars of possibly related types (late type giants, Mira variables, Algol systems, W UMa systems).

Novae and dwarf novae have similar distributions, those of fairly young stellar populations. The observed space density of potential novae (novalike objects) indicates that the mean recurrence time of novae might be as small as a few hundred years, which leads, with given nova shell masses and mass transfer rates in the minimum stage, to a secular decrease of the masses of the components undergoing nova outbursts.

Recurrent novae and symbiotic stars have distributions of older stellar populations, similar to those of late type giants and Mira variables.

On the basis of galactic distribution, novae and dwarf novae are closely related and may be final stages of W UMa systems, as well as progenitors of supernovae of type I. A small fraction of W UMa systems seems to belong to an older population. If evolutionary transitions between these types of stars can be substantiated, the presence of a minority of novae and dwarf novae in globular clusters and of supernovae I in elliptical galaxies can be explained.

Due to the lack of sufficiently well determined space distributions of Algol binaries, the suggestion that long-period Algol systems might be the progenitors of cataclysmic binaries can as yet neither be substantiated nor refuted. A very high space density of long-period Algol systems in the solar neighbourhood is derived. The observed space density of cataclysmic binaries could be explained by the transformation of a small percentage of the long-period Algol systems by common envelope evolution.
\end{abstract}

\section{Introduction}

With the advent of the space age and the possibility of studying celestial objects at a multitude of wavelengths, the interest in cataclysmic binaries (CBs) has increased, and the progress of understanding the physical processes in these systems has increased likewise. Numerous review papers dealing with various aspects of these systems and other types of binaries give an eloquent testimony of this development (for a review of reviews, see Trimble, 1983).

The determination of the evolutionary state of $\mathrm{CBs}$ receives much attention at the present time. What types of stars evolve into CBs? Where to do CBs evolve? Some outlines of binary star evolution (see, e.g., Paczynski, 1980; Vilhu, 1981; Yungelson, 1982; Eggleton, 1983) have placed the CBs into evolutionary schemes, using physical similarities of the relevant groups of stars: masses, angular momenta, periods; and plausible physical interactions of the components in the course of their evolution: Roche-lobe overflow, common envelopes, spiralling-in. Another crucial point for

* Paper presented at the Lembang-Bamberg IAU Colloquium No. 80 on 'Double Stars: Physical Properties and Generic Relations', held at Bandung, Indonesia, 3-7 June, 1983. 
establishing evolutionary relations between objects of different types is the study of their galactic distribution, and any proposed evolutionary scheme must not be in conflict with data from stellar statistics.

In this contribution, I shall review our knowledge of the galactic distribution and the space density of several types of CBs, and I shall try to compare them with data of possibly related types of binary and single stars. Since the data found in the literature are often fragmentary and imprecise, this contribution is based to a large extent on newly derived data of galactic distributions of various types of binary stars.

\section{Current Evolutionary Schemes Including CBs}

In the late 50s (Sahade, 1959) and early 60s (Kraft, 1962, 1967), the scheme

$$
\mathrm{EW} \rightarrow \mathrm{CB}
$$

was proposed (EW = W UMa type eclipsing binary; the abbreviation here also includes non-eclipsing systems). This scheme could be reconciled with the space densities, galactic distributions, space motions, and periods of these types of objects, but it proved difficult in the following decades to find a feasible mechanism for the production of CBS out of EWs. The scheme was revived and extended by Warner (1974a) to

$$
\mathrm{EW} \rightarrow \mathrm{CB} \rightarrow \mathrm{SN} \text { I . }
$$

The second half of this scheme, the production of supernovae of type I from CBs, where the mass of the white dwarf is pushed, by accretion, beyond the Chandrasekhar limit, has received much attention in recent years (e.g., Starrfield et al., 1981).

The discontinuity theory of EW structure is in favour of scheme (1) (e.g., Shu, 1980), while the thermal relaxation oscillation theory yields

$$
\mathrm{EW} \rightarrow \text { single star }
$$

(e.g., Webbink, 1976), so that another process of producing CBs must be found. One possible scheme is the evolution of a long period binary system (noted here as EA) through case $\mathrm{C}$ or case $\mathrm{B}$ evolution, including a spiralling-in of the secondary star (Paczynski, 1976; Ritter, 1976; see also Law and Ritter, 1983):

$$
\begin{aligned}
& \text { EA }(P: \text { months } \ldots \text { years }) \rightarrow \text { giant stage } \rightarrow \mathrm{CB}\left(M_{A} \geqq 0.45 M_{\odot}\right), \\
& \text { EA }(P: \text { days } \ldots \text { months }) \rightarrow \mathrm{CB}\left(M_{A} \leqq 0.45 M_{\odot}\right),
\end{aligned}
$$

where $M_{A}$ indicates the mass of the primary (white dwarf) component. We will postpone the discussion of these concurring schemes to Section 10.

\section{Methods}

Previous studies of the galactic distribution of various types of objects were based on different methods, and the parameters were defined in different ways. We have tried to 
convert the results into a set of parameters that allows intercomparison. The following quantities were collected or extracted from published data, or were newly determined by the straightforward method described by Kurkarkin (1954), using data taken from the General Catalogue of Variable Stars (CGVS) and its three supplements, unless mentioned otherwise:

$$
\begin{aligned}
& \rho_{0}=\text { space density of the objects in the galactic plane, in the neighbourhood } \\
& \text { of the Sun }\left(\mathrm{pc}^{-3}\right) ; \\
& z_{0}=\text { scale height of the distribution perpendicular to the galactic plane in the } \\
& \quad \text { solar neighbourhood, assuming a density law } \\
& \rho(z)=\rho_{0} \exp \left(-|z| / z_{0}\right) ;
\end{aligned}
$$

or

$$
\rho(z)=\rho_{0} \exp \left(-z^{2} / 2 z_{0}^{2}\right) .
$$

We have not attempted to derive the radial density gradient, $\partial \rho / \partial R$, and we have not corrected the samples for the slight displacement of the Sun from the galactic plane. Furthermore, space motions of the various groups of stars are not considered. Earlier material on space motions has already been discussed (e.g., Kraft, 1965), and more recent studies are not available. It is expected that the HIPPARCOS mission will provide data for a more detailed study of the kinematics of various stellar types.

In this text we generally discuss the parameters of approximations using form (5a). It should be noted, however, that distributions of the form (5b) can also be fitted to the data, yielding fits of similar quality. The results of the approximations of the form (5b) are given in Table IX which summarizes all results.

\section{Galactic Distribution of Novae}

The population assignment of novae has always been a matter of discussion. Baade (1958) counts them among Population II objects because of the occurrence of novae in globular clusters. Plaut (1965) emphasizes the concentration of novae towards the galactic plane and the galactic centre, stating that they form a 'disc population'. Iwanowska and Burnicki (1962) who assume that any group can be described as a mixture of Population I and Population II objects, find evidence for their assumption. Observations of novae in M31 also yield discordant results (Rosino, 1973, 'disc population'; Wenzel and Meinunger, 1978, 'halo population'). Table I summarizes the results of previous investigations.

For the rediscussion of the galactic distribution of novae, the GCVS was used as a source list. Crucial parameters for the determination of the galactic distribution and the space density of a given group of stars are:

(a) the absolute magnitude (or the magnitude range) of the stars in the group;

(b) the completeness of the search as a function of distance;

(c) the interstellar absorption. 
TABLE I

Previous determinations of the galactic distribution of novae

\begin{tabular}{llll}
\hline Author & $\rho_{0}\left(\mathrm{pc}^{-3}\right)$ & $z_{0}(\mathrm{pc})$ & Comments \\
\hline Kukarkin (1954) & - & 250 & (originally published 1949) \\
Parenago (1955) & $5.5 \times 10^{-6}$ & 109 & \\
Kopylov (1957) & - & 182 & \\
Schmidt-Kaler (1957) & - & 434 & \\
Oort (1958) & - & 440 & \\
Iwanowska and Burnicki (1962) & $\sim 10^{-7}$ & 72 & (Population I objects only) \\
Plaut (1965) & - & 290 & (source: Schmidt-Kaler (1957) (?!)) \\
Richter (1968) & - & 182 & (source: Kopylov (1957)) \\
Allen (1973) & - & 300 & \\
Warner (1974) & $10^{-8}-10^{-7}$ & - & \\
\hline
\end{tabular}

We rely on the luminosity calibration and distance determinations by Duerbeck (1981). Only novae with recorded outbursts are included, the few recurrent novae are treated separately, WZ Sge-type objects are omitted. A problem which hardly influences the $z$-distribution, but very much the space density, is that of completeness. Are all novae that erupt during a given time interval discovered? Novae that are faint during maximum light, southern novae, novae in the more distant past had a better chance to be overlooked. The seasonal variation of the night sky also plays a role, especially for fast novae. Novae that erupted between 1848 and 1981 have been included in our list, covering 133 yr of observation. But only since the beginning of the 20th century the sky has been searched in a more or less regular way, and, taking into account the seasonal effect, the 'effective' observing time is certainly closer to $50 \mathrm{yr}$, the value used in the subsequent calculations. 20 novae are found in a cylinder with a radius $d_{x y}=1500 \mathrm{pc}$ and infinite height, centered in the Sun and extending into the $z$-direction of the Galaxy, i.e., 0.40 novae per year (Table II). Novae at the edge of this cylinder reach apparent maximum magnitudes between $4^{m}$ and $5^{m}$. A study of the novae in a cylinder with $d_{x y}=1000 \mathrm{pc}$ yields a similar $z$-distribution, however, it contains more novae than expected from the ratio of the volumes of the cylinders, indicating that the discovery of novae is not complete (or, at least, was not complete in the past) to a limiting magnitude $4^{m}$ to $5^{m}$. This confirms the findings of Allen (1954) and Schmidt-Kaler (1957) that only novae with apparent magnitudes brighter than $3^{m} .0$ (distance less or equal to $1000 \mathrm{pc}$ ) were discovered with certainty (Figure 1 ). The space/time density $\rho^{*}$ and the distribution were derived from novae in the cylinder with $d_{x y}=1 \mathrm{kpc}$, yielding

$$
\rho^{*}(z)=(0.38 \pm 0.04) \times 10^{-9} \exp (-|z| /(125 \pm 22))\left(\mathrm{pc}^{-3} \mathrm{yr}^{-1}\right)
$$

(see Figure 2). The space density can be obtained when an estimate of the mean recurrence time of outbursts is available. Let us first assume that the nova phenomenon is a quasi-stationary process, i.e., the mass ejected during outburst is equal to the mass accreted between subsequent outbursts. Shell masses are estimated to lie in the range $(2-10) \times 10^{-5} M_{\odot}$ (Duerbeck, 1980). The accretion rate can be estimated from the luminosity in the minimum state. Smak (1982) has studied the energy distributions and 


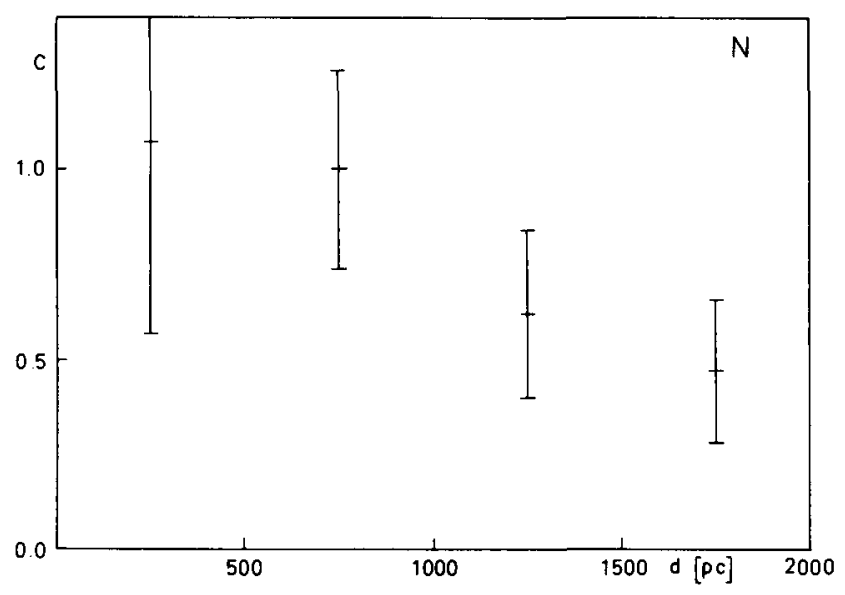

Fig. 1. Completeness of the discovery of novae in different cylinders with radii $d_{x y}$ (in pc). The completeness is set 1.0 for the cylinder with $d_{x y}=1000 \mathrm{pc}$. The completeness decreases for radii (or nova distances) which are larger than $1000 \mathrm{pc}$.

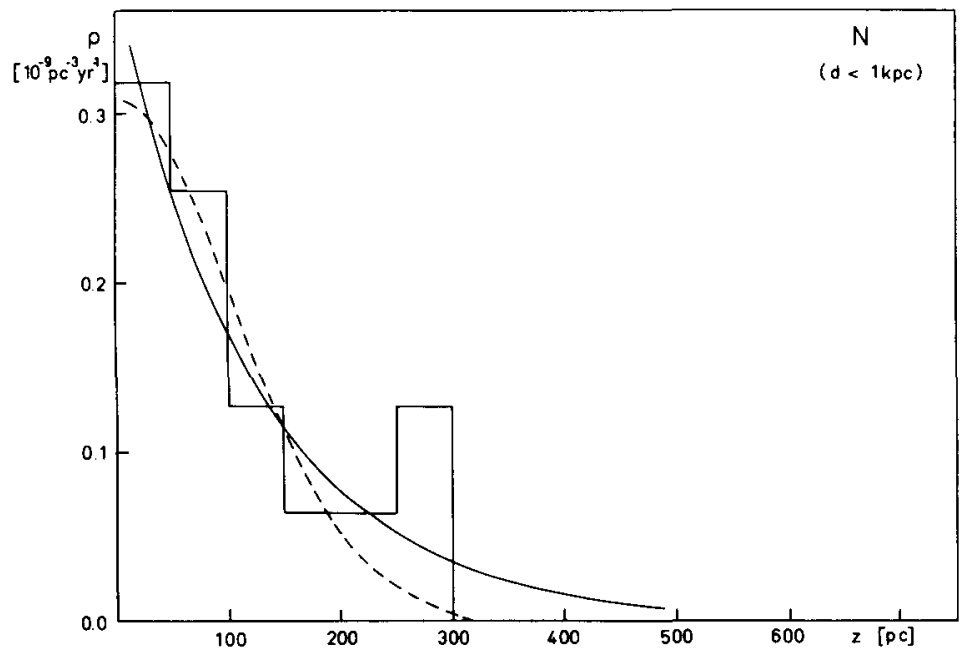

Fig. 2. The galactic distribution of novae in the $z$-direction. All novae with $d_{x y} \leqq 1000 \mathrm{pc}$ are included. The approximations of the form (5a), solid line; and (5b), broken line; are also shown.

luminosities of several novae and other CBs. His data indicate that the nova accretion rates are in the range $(0.8-3) \times 10^{-8} M_{\odot} \mathrm{yr}^{-1}$. The time interval between outbursts is then

$$
t=5 \times 10^{-5} / 1.5 \times 10^{-8}=3000 \mathrm{yr},
$$

with lower and upper limits of 700-12000 yr. The mean time interval yields a space 
density

$$
\rho_{0}=1.27 \times 10^{-6} \mathrm{pc}^{-3}
$$

for Novae.

Let us check this value. With a mean minimum magnitude $M_{V}^{\min }=4^{m} .2 \pm 1^{m} .8$ (s.d.) (Duerbeck, 1980), past and future novae, including those with unrecorded outbursts, of $m_{V} \leqq 11^{m}$ at minimum should be found in a sphere with radius $r \leqq 235 \mathrm{pc}$, under the assumption that:

(a) The brightness of novae in the long interval between outbursts does not change noticeably. This assumption can be justified by the fact that post- and pre-novae have similar magnitudes (Robinson, 1975), though some authors postulate a less active, less luminous state during most of the time between outbursts.

(b) Interstellar absorption can be neglected for distances up to $r=235 \mathrm{pc}$.

(c) The large dispersion of minimum magnitudes is not taken into account.

If we assume $M_{V}^{\text {min }}=+4^{m} .2$ for all novae, the number of novae in the minimum state with apparent magnitudes brighter than $11^{\mathrm{m}}$ can be calculated, using the density distribution function $(5 \mathrm{a})$ :

$$
N(r)=2 \pi \rho_{0}\left(z_{0} r^{2}+2 z_{0}^{2} r e^{-r / z_{0}}+2 z_{0}^{3}\left(e^{-r / z_{0}}-1\right)\right) ;
$$

or, with (5b),

$$
N(r)=2 \pi \rho_{0} r^{3} \sum_{n=0}^{\infty} \frac{1}{n !}\left(\frac{-r^{2}}{2 z_{0}^{2}}\right)^{n}(1 /(2 n+1)-1 /(2 n+3)) .
$$

Inserting the above data, we obtain in both cases,

$$
N(r \leqq 235 \mathrm{pc})=38 \text {. }
$$

Thus, there should be about 38 potential novae brighter than $11^{m}$. None of the novae observed during outburst is inside this region. Known novalike objects in the appropriate magnitude range are TT Ari (only at maximum!), V3885 Sgr, RW Sex, and CPD $-48^{\circ} 1577$ (Ritter, 1983; Garrison et al., 1982). It is open to discussion whether all these objects are potential novae.

A few novae at minimum show dwarf-nova-like light variations - e.g., GK Per (Hudec, 1981), WY Sge (Moffat, 1982), or V3890 Sgr (Dinerstein and Hoffleit, 1973). Thus some systems with unrecorded outbursts might well be classified as dwarf novae, they have, however, higher luminosities than ordinary dwarf novae. Since this class seems to constitute a small percentage of the novae, potential novae among the catalogued dwarf novae will not significantly make up the deficit. In any case, the observed space density is possibly of the order of 10 times smaller than the expected one.

Let us check the parameters which enter the determination of space density for their probable errors:

(a) The 'effective' time interval of nova observations, $50 \mathrm{yr}$, can hardly be in error by more than a factor 2 . 
(b) The accretion rates may be variable. A secular change of the accretion rate would, however, hardly improve the situation: lowering by a factor 10 would increase the time between outbursts by a factor 10 , but the minimum magnitude would also decline, by about $2^{m} .5$. Thus only novae with $r \leqq 74 \mathrm{pc}$ would be brighter than $11^{m}$. Then

$$
N(r \leqq 74 \mathrm{pc})=20,
$$

which is still to large. The minimum magnitude of novae has only a weak influence of their space density.

(c) The mean shell masses might be lower than $5 \times 10^{-5} M_{\odot}$ (contrary to observational evidence), or the nova eruptions cause a secular decrease of the mass of the erupting star, i.e., the eruption not only removes the accreted mass, but also genuine material of the white dwarf, which must then comprise a large fraction of the shell mass. In both cases, the mean recurrence time of nova eruptions would only be a few hundred years. It might be rewarding to review old written sources for probable earlier outbursts of bright novae.

TABLE II

Galactic novae with $d_{x y}<1.5 \mathrm{kpc}$

\begin{tabular}{lccrlrrr}
\hline Nova & \multicolumn{1}{l}{$b$} & $d_{x y}(\mathrm{pc})$ & $z(\mathrm{pc})$ & Nova & \multicolumn{1}{c}{$b$} & $d_{x y}(\mathrm{pc})$ & $z(\mathrm{pc})$ \\
\hline V603 Aql & +0.84 & 330 & 5 & DI Lac & -4.86 & 895 & 26 \\
T Aur & -1.71 & 600 & 18 & DK Lac & -5.35 & 1495 & 140 \\
Q Cyg & -7.55 & 1485 & 200 & BT Mon & -2.63 & 1000 & 46 \\
V1500 Cyg & -0.07 & 1350 & 2 & V841 Oph & +17.79 & 855 & 275 \\
HR Del & -13.97 & 775 & 212 & GK Per & -10.11 & 515 & 92 \\
DN Gem & +14.70 & 435 & 114 & RR Pic & -25.67 & 360 & 173 \\
DQ Her & +26.44 & 235 & 116 & CP Pup & -0.84 & 1500 & 22 \\
V446 Her & +4.71 & 785 & 65 & FH Ser & +5.78 & 645 & 65 \\
V533 Her & +24.27 & 620 & 280 & LV Vul & +0.85 & 820 & 12 \\
CP Lac & -0.84 & 1000 & 15 & NQ Vul & +1.28 & 1200 & 27 \\
\hline
\end{tabular}

In the discussion concerning secular mass loss the recurrent nova $\mathrm{T}$ Pyx may play an important role. It is the only slow recurrent nova $\left(t_{3}=113^{\mathrm{d}}\right)$. Its spectral appearance at outburst, which is similar to that of normal novae, its fairly strong surrounding nebulosity (Duerbeck and Seitter, 1979), its blue colour at minimum light (Bruch et al., 1981) separate it from the properties of all other recurrent novae, and link it to the group of ordinary slow novae. The only peculiarity is the outburst interval (about $19 \pm 5 \mathrm{yr}$ ) which cannot be reconciled with any reasonable accretion rate if steady state nova evolution is assumed. A depletion of the mass of the accreting object, as indicated by our statistical study of all novae, would resolve the problem. A thorough investigation of this unique object, which is likely to show another outburst soon, might considerably improve our knowledge of evolutionary trends in nova systems.

Taking into account the above mentioned ambiguities, the space density (8) should be taken as an upper limit; there is evidence that $\rho_{0}$ may be smaller by at least a factor 10 . 


\section{Galactic Distribution of Recurrent Novae}

The group of recurrent novae is small and inhomogeneous. A recent list can be found in Duerbeck and Seitter (1982). It is still subject to discussion whether their light eruptions are thermonuclear runaways or accretion events (Webbink, 1978). Each member of the group shows characteristics that make its memberhsip uncertain:

(a) $\mathrm{T} \mathrm{CrB}$ can be explained as an accretion event onto a non-degenerate star (Webbink, 1978);

(b) VY Aqr has a very blue colour; evidence for a fairly short outburst interval (Richter, 1983) make its membership in the group of dwarf novae likely;

(c) WZ Sge, showing typical dwarf nova characteristics during outburst, has already been removed from the group;

(d) T Pyx, the only 'slow' recurrent nova, has certainly no giant secondary, however, it has a noticeable shell, thus it cannot be a dwarf nova (see Section 4);

(e) U Sco possibly has also a dwarf companion (Barlow et al., 1981);

(f) V1017 Sgr has been classified as a symbiotic star by Payne-Gaposchkin (1977), however, the observed 'secondary maxima' have some resemblance to the photometric behaviour of T CrB (Vidal and Rodgers, 1974).

An attempt was made to obtain at least a rough estimate of $z_{0}$. Distances were derived under the assumption that the spectroscopically detectable secondaries are giant (or dwarf) stars; the result for $\mathrm{T} \mathrm{CrB}, r=1200 \mathrm{pc}$, is in good agreement with the nebular parallax (Duerbeck, 1981). For U Sco, a dwarf secondary of spectral type $G-K$ was assumed. Details are given in Table III.

TABLE III

List of recurrent novae

\begin{tabular}{lrrlllllc}
\hline Object & $b$ & $m_{V}(\mathrm{~min})$ & \multicolumn{1}{l}{$A_{V}$} & \multicolumn{2}{l}{ Spectrum } & \multicolumn{1}{l}{$M_{V}$} & $d(\mathrm{pc})$ & $z(\mathrm{pc})$ \\
\hline T CrB & +48.2 & $9^{m} .9(1)$ & $0^{m} \cdot 1(2)$ & M3 III & $(3)$ & $-0^{m} .6$ & 1200 & 300 \\
RS Oph & +10.4 & $11.4(1)$ & $2.4(2)$ & M2 III/A7Q(3) & -0.6 & 1800 & 325 \\
U Sco & +21.9 & $19.0(2)$ & $0.6(2)$ & G0-K0V? & $(4)$ & $4.5-6.0$ & $3-6 \times 10^{3}$ & $1100-2200$ \\
V1017 Sgr & -9.1 & $13.6(1)$ & $1.0(2)$ & G5 III p & $(3)$ & +0.9 & 2200 & 350 \\
\hline
\end{tabular}

References: (1) Bruch (1982); (2) Webbink (1978); (3) Duerbeck and Seitter (1982); (4) Barlow et al. (1981).

TABLE IV

Previous determinations of the galactic distribution of recurrent novae

\begin{tabular}{llll}
\hline Author & $\rho_{0}\left(\mathrm{pc}^{-3}\right)$ & $z_{0}(\mathrm{pc})$ & Comments \\
\hline Kopylov (1957) & - & 480 & \\
Richter (1968) & - & 480 & (source: Kopylov (1957)) \\
Allen (1973) & - & 500 & \\
Warner (1974) & $8 \times 10^{-10}$ & - & \\
\hline
\end{tabular}


The resulting mean $z_{0}, 660 \pm 330 \mathrm{pc}$, is not unlike that of symbiotic stars (see Section 7), and very different from that of classical novae and dwarf novae. Previously derived data are summarized in Table IV.

\section{Galactic Distribution of Dwarf Novae}

Previous estimates of the scale height of dwarf novae vary noticeably (Table V). We have therefore determined the distribution of dwarf novae again.

TABLE V

Previous determinations of the galactic distribution of dwarf novae

\begin{tabular}{lccl}
\hline Author & $\rho_{0}\left(\mathrm{pc}^{-3}\right)$ & $z_{\mathbf{0}}(\mathrm{pc})$ & Comments \\
\hline Kopylov (1957) & - & 2600 & \\
Schmidt-Kaler (1957) & - & 90 & \\
Kraft (1962) & - & $48^{*}$ & \\
Popov (1964) & $\sim 10^{-5 *}$ & $63 / 39^{*}$ & \\
Kraft (1965) & - & $60 / 84^{*}$ & \\
Richter (1968) & $5 \times 10^{-6}$ & 111 & (also $z_{0}=2600$ quoted) \\
Warner (1974) & $4 \times 10^{-7}$ & - & \\
\hline
\end{tabular}

* Values marked with an asterisk were derived from the original data, using approximations (5a) and (5b).

Of the $\sim 300$ dwarf novae listed in the GCVS and its supplements, 72 are found in a cylinder of $400 \mathrm{pc}$ radius and infinite height. The crucial parameter that determines the derived distribution is the absolute magnitude. Different statistical studies yield for the absolute magnitude at minimum, $\left\langle M_{V}^{\text {min }}\right\rangle$, values around $+7^{m} \cdot 5$ (Kraft and Luyten, 1965; Primkulov, 1968; Voikhanskaya, 1973). However, trigonometric parallaxes of several dwarf novae yield values of $\left\langle M_{V}^{\min }\right\rangle=9^{m} .5 \pm 1^{m} .2$ (s.d.) (Vasilevskis et al., 1975). A recent discussion of the parallaxes of SS Cyg, U Gem, and SS Aur by Kamper (1979) yields $\left\langle M_{V}^{\min }\right\rangle=+8^{m} \cdot 9 \pm 0.8$ and $\left\langle M_{V}^{\max }\right\rangle=4^{m} \cdot 4 \pm 1^{m} \cdot 3$. A study based on $K$-line photometry of four objects by Bailey (1981) yields $\left\langle M_{V}^{\min }\right\rangle=+8^{m} \cdot 5 \pm 1^{m} \cdot 9$ and $\left\langle M_{V}^{\max }\right\rangle=+4^{m} \cdot 4 \pm 1^{m} .1$. Vogt (1981) showed that the luminosities of the discs of all dwarf novae during outburst seems to be equal, corresponding to $\left\langle M_{V}^{\max }\right\rangle=+4^{m} .7$. The mean outburst amplitude of all dwarf novae for which reliable data exist is $3^{m}$. 9 magnitudes. The old assumption, $\left\langle M_{V}^{\min }\right\rangle=+7^{m} \cdot 5$, gives $\left\langle M_{V}^{\max }\right\rangle=+3^{m} \cdot 6$, a brightness that is much higher than the recently obtained results.

We have adopted for the following discussing

$$
\left\langle M_{V}^{\max }\right\rangle=+4^{m} \cdot 5
$$

corresponding to $\left\langle M_{V}^{\min }\right\rangle=+8^{m} \cdot 4$. It is advantageous to use the brightness at maximum, because it has probably a lower intrinsic scatter, and is better documented.

The 44 objects that are included in a cylinder of $250 \mathrm{pc}$ radius are listed in Table VI, where distances and $z$-values are given. The absorption was estimated by using the study 
TABLE VI

Dwarf novae with $d_{x y}<250 p c$

\begin{tabular}{|c|c|c|c|c|c|}
\hline Object & $b$ & $m_{V}^{\max }$ & Type & $d_{x y}(\mathrm{pc})$ & $z(\mathrm{pc})$ \\
\hline RX And & -21.5 & 10.3 & $\mathrm{ZC}$ & 135 & 53 \\
\hline DX And & -16.7 & 11.2 & UG & 210 & 62 \\
\hline Z Aps & -9.4 & 10.7 & $\mathrm{ZC}$ & 175 & 29 \\
\hline VZ Aqr & -36.3 & 11.6 & UG & 195 & 142 \\
\hline UU Aql & -18.8 & 11.3 & UG & 220 & 66 \\
\hline SS Aur & +13.8 & 10.5 & UG & 155 & 38 \\
\hline TT Boo & +60.7 & 12.7 & UG & 215 & 379 \\
\hline Z Cam & +32.6 & 10.2 & $\mathrm{ZC}$ & 120 & 75 \\
\hline SY Cnc & +36.4 & 10.9 & $\mathrm{ZC}$ & 155 & 113 \\
\hline AA Cnc & +33.9 & 11.8 & UG? & 240 & 159 \\
\hline BV Cen & +7.5 & 10.7 & UG & 160 & 21 \\
\hline WW Cet & -71.7 & 9.6 & $\mathrm{ZC}$ & 31 & 95 \\
\hline WX Cet & -79.1 & 10.8 & WZ & 35 & 172 \\
\hline Z Cha & -22.1 & 11.5 & $\mathrm{UG} / \mathrm{SU}$ & 220 & 90 \\
\hline AL Com & +76.4 & 13.3 & $w Z$ & 135 & 559 \\
\hline GO Com & +88.7 & 13.4 & WZ & 15 & 605 \\
\hline SS Cyg & -7.1 & 8.2 & UG & 55 & 6 \\
\hline SY For & -62.4 & 12.3 & UG & 145 & 279 \\
\hline U Gem & +23.4 & 8.2 & UG & 50 & 22 \\
\hline IR Gem & +11.6 & 10.7 & UG & 145 & 30 \\
\hline AH Her & +38.2 & 10.5 & $\mathrm{ZC}$ & 125 & 96 \\
\hline EX Hya & +33.6 & 11.7 & UG & 230 & 152 \\
\hline VW Hyi & -38.1 & 8.4 & $\mathrm{UG} / \mathrm{SU}$ & 45 & 37 \\
\hline WX Hyi & -51.6 & 9.6 & UG/SU & 65 & 82 \\
\hline T Leo & +60.5 & 10.3 & UG & 70 & 126 \\
\hline X Leo & +45.1 & 11.1 & UG & 150 & 149 \\
\hline TU Leo & +44.0 & 12.0 & UG & 225 & 219 \\
\hline TU Men & -33.7 & 11.7 & UG/SU? & 230 & 152 \\
\hline CN Ori & -15.6 & 11.5 & $\mathrm{ZC}$ & 225 & 63 \\
\hline RU Peg & -34.8 & 9.0 & UG & 60 & 41 \\
\hline EZ Peg & -32.5 & 9.8 & UG? & 95 & 59 \\
\hline FO Peg & -0.5 & 11.8 & $\mathrm{ZC}$ & 250 & 2 \\
\hline KT Per & -11.3 & 11.0 & $Z C$ & 155 & 31 \\
\hline WZ Sge & -7.9 & 7.3 & $\mathrm{WZ}$ & 35 & 5 \\
\hline $\mathrm{V} 1830 \mathrm{Sgr}$ & -4.8 & 11.8 & UG & 245 & 21 \\
\hline UZ Ser & +1.8 & 12.3 & UG & 250 & 8 \\
\hline EK TrA & -6.3 & 12.2 & $\mathrm{UG} / \mathrm{SU}$ & 240 & 26 \\
\hline SU UMa & +33.1 & 11.0 & $\mathrm{UG} / \mathrm{SU}$ & 170 & 109 \\
\hline SW UMa & +37.0 & 11.1 & UG & 170 & 126 \\
\hline BC UMa & +65.1 & 11.2 & UG? & 95 & 199 \\
\hline BZ UMa & +38.8 & 10.8 & UG? & 140 & 113 \\
\hline $\mathrm{CH} \mathrm{UMa}$ & +42.6 & 11.0 & $\mathbf{U G}$ & 145 & 135 \\
\hline CU Vel & +2.6 & 11.0 & $\mathrm{UG} / \mathrm{SU}$ & 190 & 9 \\
\hline TW Vir & +54.6 & 12.1 & UG & 190 & 269 \\
\hline
\end{tabular}


of the distribution of interstellar extinction by Neckel (1967), and, for stars near the galactic equator, by Neckel and Klare (1980).

It is important to find out the limiting distance for which completeness of the sample is guaranteed. The surface density $\Sigma_{\mathrm{DN}}$,

$$
\Sigma_{\mathrm{DN}}=2 \rho_{0, \mathrm{DN}} \mathbf{z}_{0, \mathrm{DN}}
$$

was determined for cylinders with various radii $d_{x y}$. If we assume $\Sigma_{\mathrm{DN}}=1$ for the cylinder with $d_{x y}=200 \mathrm{pc}$, the relative surface density drops noticeably for cylinders with $d_{x y}>250 \mathrm{pc}$, that is, our knowledge of these systems is incomplete beyond $250 \mathrm{pc}$, corresponding $m_{V}^{\max }=11^{m} .5$ (Figure 3 ). The $z$-distribution in the cylinder with

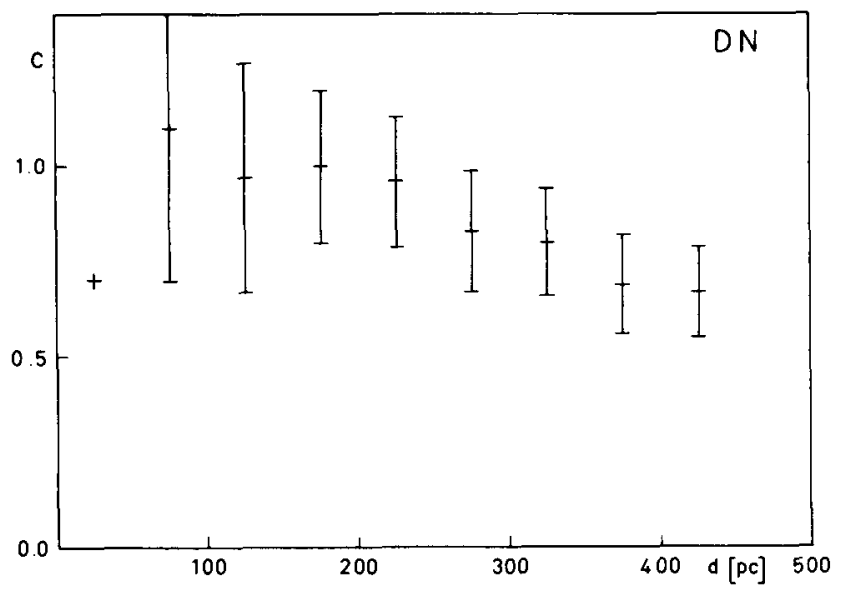

Fig. 3. Completeness of the discovery of dwarf novae in different cylinders with radii $d_{x y}$ (in pc). The completeness is set to 1.0 for the cylinder with $d_{x y}=200 \mathrm{pc}$. The completeness decreases slowly for radii which are larger than $250 \mathrm{pc}$.

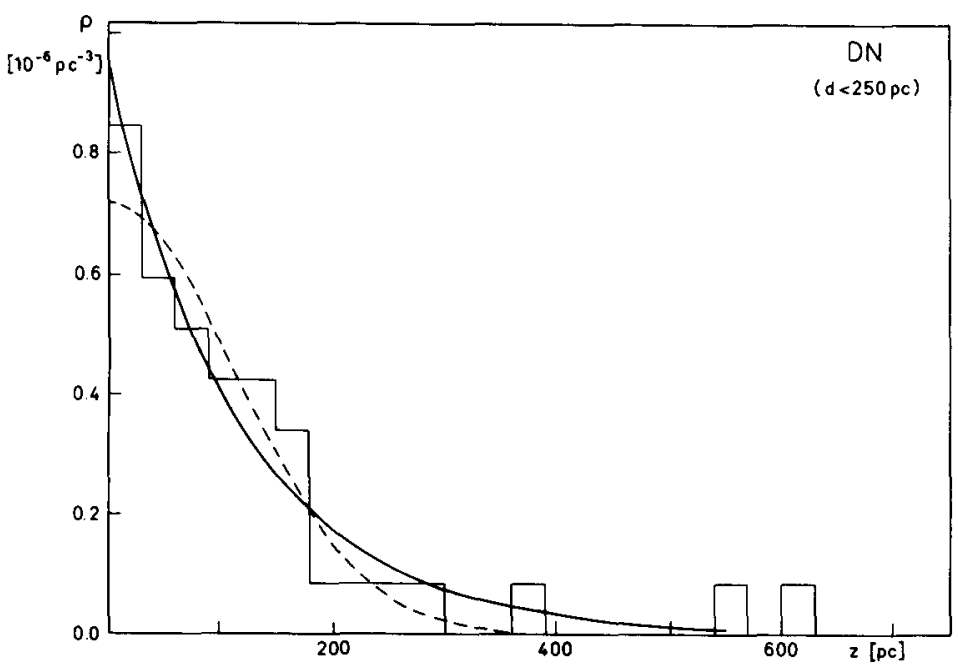

Fig. 4. The galactic distribution of dwarf novae in the $z$-direction. All dwarf novae with $d_{x y} \leqq 250 \mathrm{pc}$ are included. 
$d_{x y}=250 \mathrm{pc}$ was determined. It can be approximated by

$$
\rho(z)=(0.95 \pm 0.05) \times 10^{-6} \exp (-|z| /(119 \pm 9)) \quad\left(\mathrm{pc}^{-3}\right)
$$

(Figure 4). Since the value of $z_{0}$ is well determined by the objects with $z$-distances up to $250 \mathrm{pc}$, it does not have to be corrected for incompleteness.

\section{Galactic Distribution of Symbiotic Stars}

The catalogue of symbiotic stars by Allen (1979) wa used; the distance estimates were taken from Allen (1980). More than most other groups studied here, the group of symbiotic stars suffers from small number statistics. The completeness and distribution in cylinders with $d_{x y}=1,2,3$, and $4 \mathrm{kpc}$ was studied (Figure 5). The results for $d_{x y}=2$

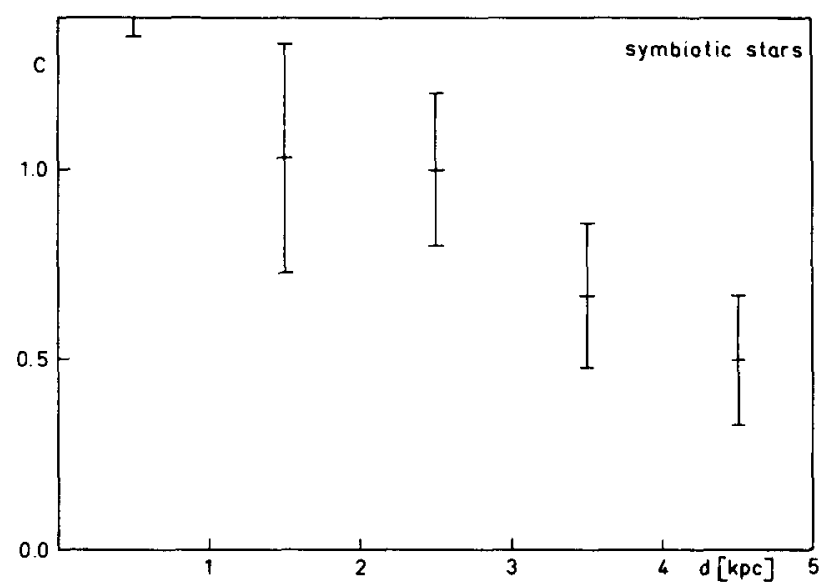

Fig. 5. Completeness of the discovery of symbiotic stars in different cylinders with radii $d_{x y}$ (in kpc). The completeness is set to 1.0 for the cylinder with $d_{x y}=3 \mathrm{kpc}$. Besides a high number of symbiotic stars in the solar vicinity $\left(d_{x y} \leqq 1 \mathrm{kpc}\right)$, the completeness seems to be constant between 2 and $3 \mathrm{kpc}$ decreases at larger distances.

and $3 \mathrm{kpc}$ are quite similar, and they are probably not yet strongly affected by the interstellar extinction in the galactic plane. The distribution of the 24 symbiotic stars with $d_{x y} \leqq 3 \mathrm{kpc}$ can be approximated by

$$
\rho(z)=(9.4 \pm 2.2) \times 10^{-10} \exp (-|z| /(565 \pm 230)) \quad\left(\mathrm{pc}^{-3}\right)
$$

(see Figure 6). The population is obviously an old disc population, as was pointed out by Wallerstein (1981) on kinematical grounds, and by Allen (1980), who used the same material as we did.

\section{Galactic Distribution of Supernovae}

No own investigation was carried out. Table VII summarizes the results of different authors. 


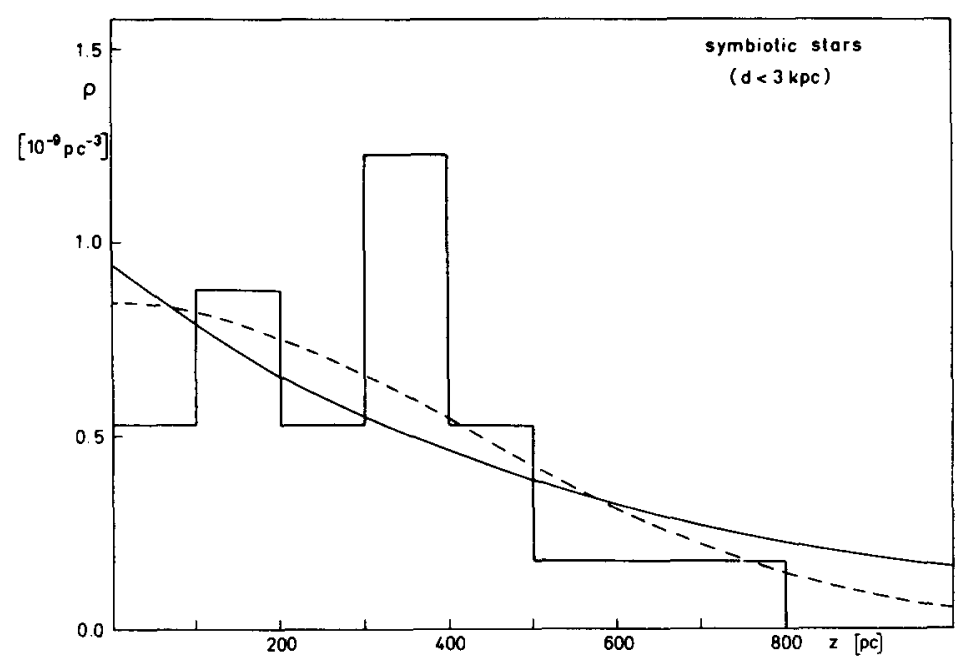

Fig. 6. The galactic distribution of symbiotic stars in the $z$-direction. All symbiotic stars with $d_{x y} \leqq 3 \mathrm{kpc}$ are included.

TABLE VII

Previous determinations of the galactic distribution of supernovae

\begin{tabular}{llrl}
\hline Author & $\rho_{0}\left(\mathrm{pc}^{-3}\right)$ & $z_{0}(\mathrm{pc})$ & Comments \\
\hline Kopylov (1957) & - & 83 & \\
Johnson and MacLeod (1963) & - & 135 & (type I) \\
& & 68 & (type II) \\
Richter (1968) & - & 83 & (source: Kopylov (1957)) \\
Tsvetkov (1981) & - & 100 & (type I and II) \\
& & &
\end{tabular}

\section{Other Types of Binaries and Single Stars}

\subsection{W UMa SYSTEMS (EW)}

In the past, CBs were thought to be descendants of EWs; more recently, this scheme is treated with more reservation because of major theoretical difficulties. We thought, however, that it might be useful to include a study of the galactic distribution of EWs, since more data are now available, as compared with the first comparative studies (Kraft, 1962, 1965; Popov, 1964).

The GCVS and the Finding List for Observers of Interacting Binaries were consulted. It was assumed that systems with known spectral types have luminosities equal to those of Main-Sequence stars of the same spectral types. The luminosity calibration of Schmidt-Kaler (1982) was used. The period is the only known parameter of systems for which no spectral type is available. A period - spectral type relation, similar to the well-known period - colour relation, was established from the data of well-investigated 
systems and was used for a coarse determination of the luminosities of poorly known systems.

Systems in a cylinder with $d_{x y}=110 \mathrm{pc}$ were used for the derivation of the $z$-distribution, which can be approximated by

$$
\rho(z)=(10.7 \pm 0.7) \times 10^{-6} \exp (-|z| /(76 \pm 6)) \quad\left(\mathrm{pc}^{-3}\right)
$$

(see Figure 7). This is, however, the space density of discovered EW systems. About $\frac{2}{3}$ of the systems remain undiscovered due to the lack of eclipses. The true space density is about three times as high, and the $z$-distribution remains the same only under the assumption that there is no preferential orientation of orbital planes (e.g., parallel to the plane of the Galaxy).

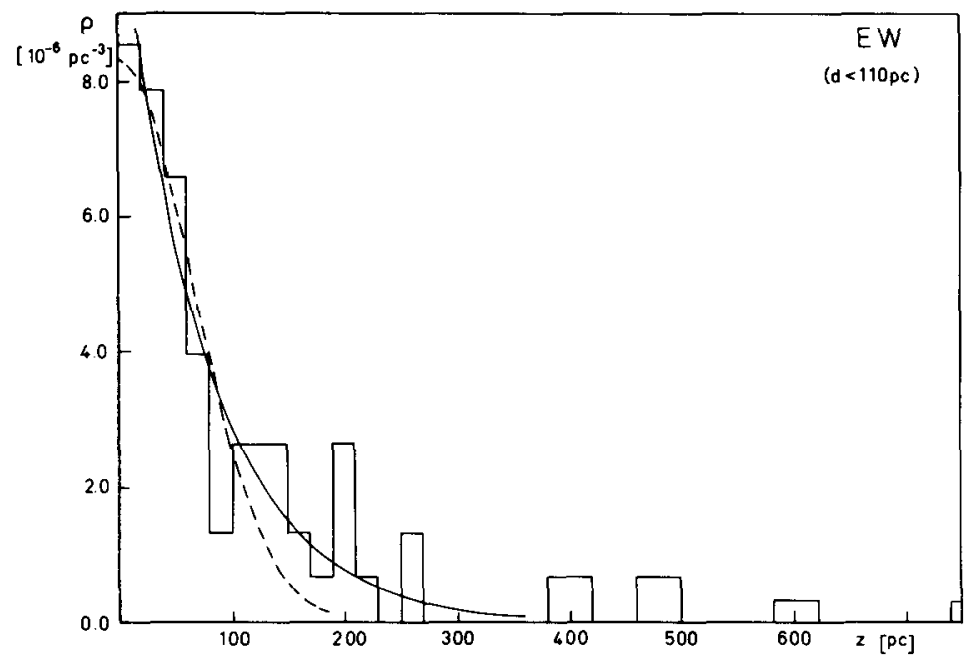

Fig. 7. The galactic distribution of W UMa type binaries in the $z$-direction. All systems with $d_{x y} \leqq 110 \mathrm{pc}$ are included.

It should also be noted that the omission of EW systems with periods $P>0.5$ days leads to a somewhat different distribution:

$$
\rho(z)=(7.5 \pm 0.5) \times 10^{-6} \exp (-|z| /(96 \pm 10)) \quad\left(\mathrm{pc}^{-3}\right) .
$$

Earlier determinations are summarized in Table VIII.

Van 't Veer's (1975) claim of a short lifetime for the EW systems is in good agreement with the small $z$-value determined above. However, when the distribution (Figure 7) is inspected more closely, a small percentage of old systems seems to be present at high galactic z-distances. The systems VW CVn, VY Cet, SS Com, AQ Com, DD Com, EK Com, BL Leo, AD Phe, and SZ Scl are objects with $z>200 \mathrm{pc}$. Not all of them have been investigated thoroughly so far, but at least half of them have published or unpublished light curves (Hoffmann, 1983), thus a confusion with RRc variables can be excluded. It would certainly be rewarding to carry out a comparative study of these 
TABLE VIII

Previous determinations of the galactic distribution of W UMa systems

\begin{tabular}{|c|c|c|c|}
\hline Author & $\rho_{0}\left(\mathrm{pc}^{-3}\right)$ & $z_{0}(\mathrm{pc})$ & Comments \\
\hline Kraft (1962) & - & $60^{*}$ & Improved by Kraft (1965) \\
\hline Popov (1964) & $\sim 2 \times 10^{-5 *}$ & $144^{*}$ & \\
\hline Kraft (1965) & - & $62 / 87^{*}$ & \\
\hline Kraft (1967) & $10^{-6}$ & - & $0.2 \%$ of $\mathrm{G}$ stars \\
\hline \multirow[t]{3}{*}{$\operatorname{van}{ }^{\prime} t$ Veer $(1975)^{a}$} & $3.6 \times 10^{-5}$ & - & Spectral type $F ; 0.8 \%$ of $F$ stars \\
\hline & $7.5 \times 10^{-5}$ & - & Spectral type $\mathrm{G} ; 1.2 \%$ of $\mathrm{G}$ stars \\
\hline & $11 \times 10^{-5}$ & - & All types \\
\hline
\end{tabular}

* Values marked with an asterisk were derived from the original data, using approximations (5a) and (5b).

a van 't Veer (1975) gives percentages of W UMa systems among the F- and G-type Main-Sequence stars; these were converted to absolute space densities, using Upgren's (1963) space densities of Main-Sequence stars.

systems with those in the solar neighbourhood, to find out whether ageing effects are present or whether these systems evolved into contact only a short time ago.

\subsection{ALGOL SYSTEMS AND OTHER BINARIES OF LONG PERIOD}

In recent theoretical studies, long period Algol systems are regarded as the most probable progenitors of CBs. Systems listed in the GCVS and the Finding List were examined. Data for systems with periods $P<1.5,1.5<P<5^{\mathrm{d}}$, and $P>5^{\mathrm{d}}$ and spectral types of the primaries earlier than F5 were analyzed separately. Distances were calculated under the assumption that the primaries are Main-Sequence stars (unless other information was supplied by the catalogues). The galactic $z$-distributions of the

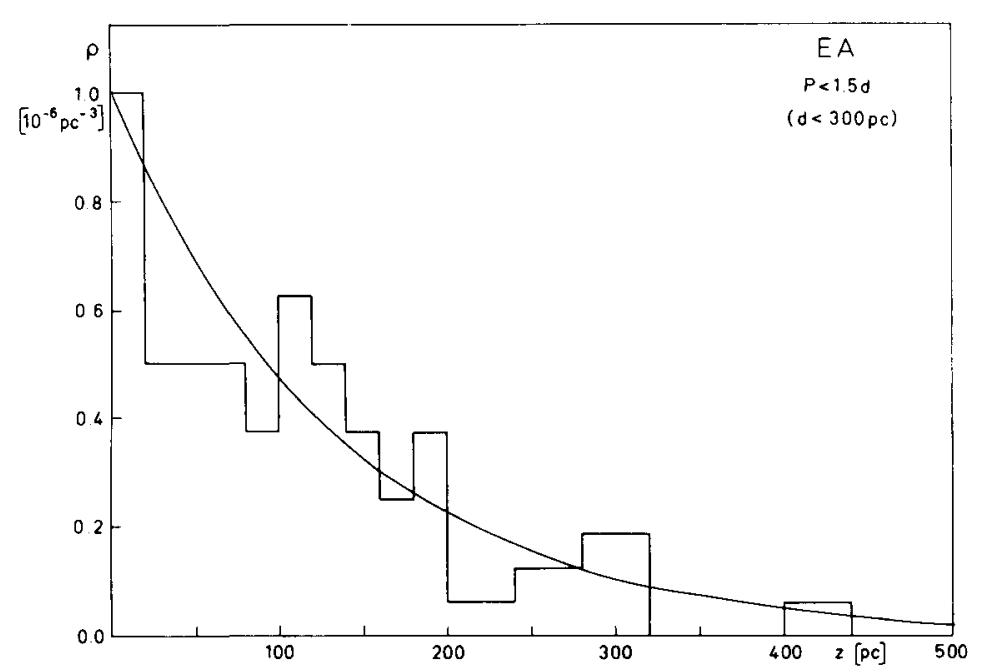

Fig. 8. The galactic distribution of Algol type binaries in the $z$-direction. Systems with $P<1.5$ days and $d_{x y} \leqq 300 \mathrm{pc}$ are included. 
systems in cylinders with $d_{x y} \leqq 100,200$, and $300 \mathrm{pc}$ are poorly defined, and the space densities decrease noticeably when the larger volumes are considered. Rough estimates, which should be treated with some reservation, are given in Table IX (see also Figures 8, 9 , and 10).

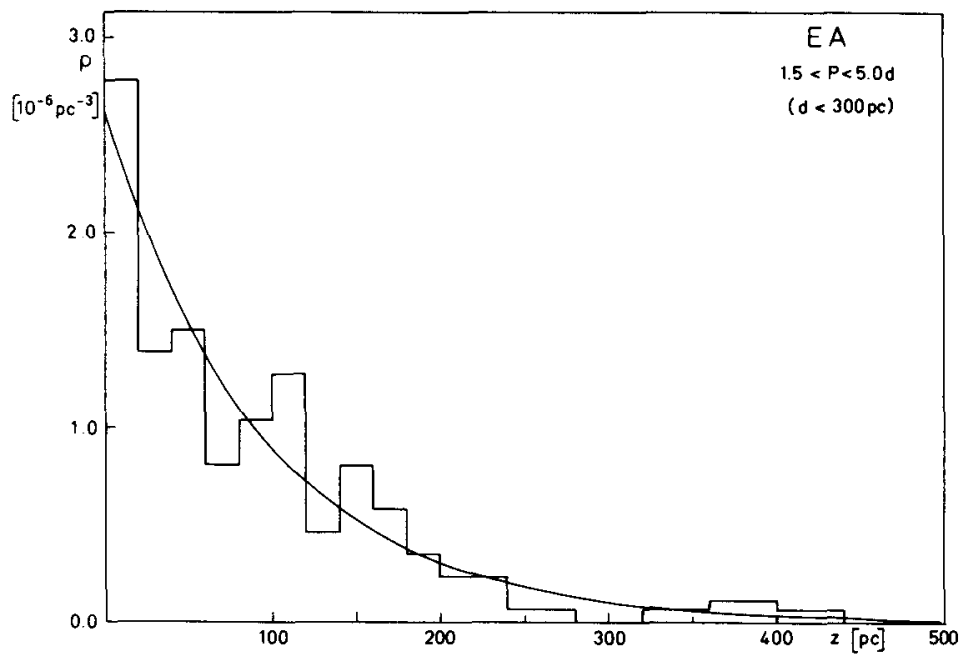

Fig. 9. The galactic distribution of Algol type binaries in the $z$-direction. Systems with 1.5 days $<P<5$ days and $d_{x y} \leqq 300$ pc are included.

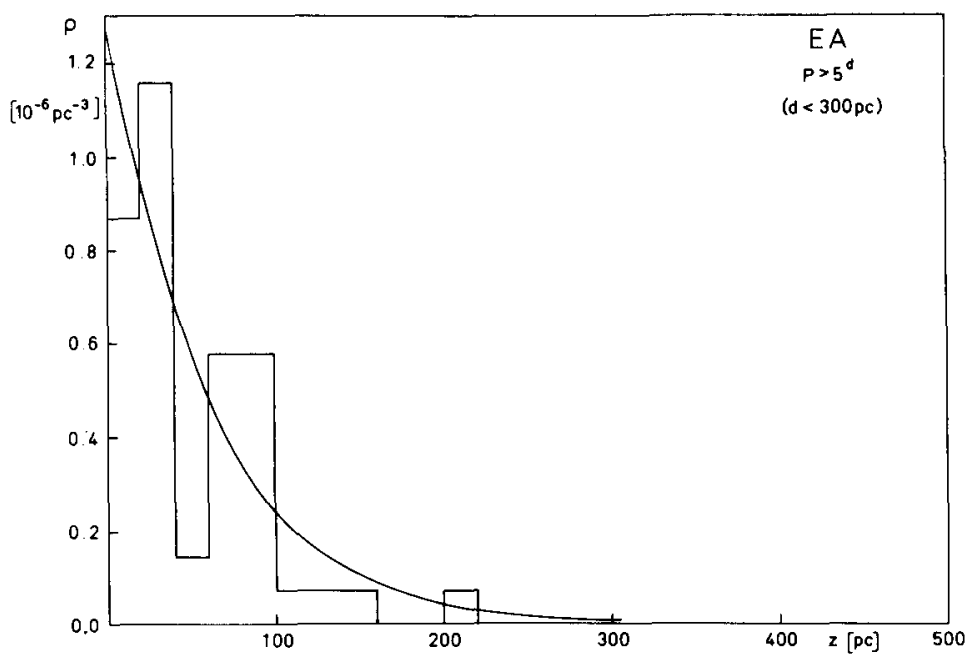

Fig. 10. The galactic distribution of Algol type binaries in the $z$-direction. Systems with $P>5$ days and $d \leqq 300$ pc are included.

More than in the previous sections, selection effects play a decisive role, because only systems showing eclipse effects are catalogued. The space densities listed in Table IX have not been corrected for the probability of discovery, and are crude lower limits of 
TABLE IX

Galactic distribution of cataclysmic binaries and possibly related types

\begin{tabular}{|c|c|c|c|c|c|}
\hline Group & $\rho_{0}\left(\mathrm{pc}^{-3}\right)$ & $z_{0}(\mathrm{pc})$ & $\rho_{0}^{\prime}\left(\mathrm{pc}^{-3}\right)$ & $z_{0}^{\prime}(\mathrm{pc})$ & Comments/reference \\
\hline $\mathrm{N}$ & $(0.38 \pm 0.04) \times 10^{-9}$ & $125 \pm 22$ & $(0.31 \pm 0.04) \times 10^{-9}$ & $107 \pm 15$ & novae per year \\
\hline RN & $(0.8) \times 10^{-9}$ & $660 \pm 330$ & & & Warner (1974) \\
\hline DN & $(0.95 \pm 0.05) \times 10^{-6}$ & $119 \pm 9$ & $(0.72 \pm 0.03) \times 10^{-6}$ & $114 \pm 6$ & \\
\hline $\mathrm{SN}$ & & 135 & & & Johnson and MacLeod (1963) \\
\hline Symb. & $(0.94 \pm 0.22) \times 10^{-9}$ & $565 \pm 230$ & $(0.84 \pm 0.14) \times 10^{-9}$ & $425 \pm 85$ & \\
\hline EW (all) & $(10.7 \pm 0.7) \times 10^{-6}$ & $76 \pm 6$ & $(8.3 \pm 0.5) \times 10^{-6}$ & $65 \pm 5$ & uncorrected \\
\hline $\mathrm{EW}(P<0 \mathrm{~d} 5)$ & $(7.5 \pm 0.5) \times 10^{-6}$ & $96 \pm 10$ & $(5.5 \pm 0.3) \times 10^{-6}$ & $93 \pm 7$ & uncorrected \\
\hline EA $(P<1$ d 5$)$ & $(1.0+0.2) \times 10^{-6}$ & $135+20$ & & & uncorrected \\
\hline $\mathrm{EA}\left(P<5^{\mathrm{d}}\right)$ & $(2.2+0.3) \times 10^{-6}$ & $95 \pm 15$ & & & uncorrected \\
\hline $\mathrm{EA}\left(P>5^{\mathrm{d}}\right)$ & $(1.3 \pm 0.3) \times 10^{-6}$ & $60 \pm 15$ & & & uncorrected \\
\hline M I & & & $(54 \pm 3) \times 10^{-9}$ & $213 \pm 35$ & Mikami and Ishida (1981) \\
\hline K0-K4 III & & & $(40+3) \times 10^{-6}$ & $170+17$ & Mikami and Ishida (1981) \\
\hline K5-M1 III & & & $(3.5 \pm 0.4) \times 10^{-6}$ & $202 \pm 5$ & Mikami and Ishida (1981) \\
\hline M2-M4 III & & & $(3.1 \pm 0.3) \times 10^{-6}$ & $313 \pm 11$ & Mikami and Ishida (1981) \\
\hline M5-M6 III & & & $(0.9 \pm 0.1) \times 10^{-6}$ & $392 \pm 15$ & Mikami and Ishida (1981) \\
\hline M7-M10 III & & & $(0.6 \pm 0.2) \times 10^{-6}$ & $382 \pm 14$ & Mikami and Ishida (1981) \\
\hline $\operatorname{Mira}(S, C)$ & $(25) \times 10^{-9}$ & $150 \pm 25$ & & & Richter (1968) \\
\hline Mira $\left(P>400^{\mathrm{d}}\right)$ & $(12.5 \pm 7.5) \times 10^{-9}$ & $300 \pm 70$ & & & Richter (1968) \\
\hline Mira $\left(P>350^{\mathrm{d}}\right)$ & $(20 \pm 10) \times 10^{-9}$ & $300 \pm 30$ & & & Richter (1968) \\
\hline Mira $\left(P>300^{\mathrm{d}}\right)$ & $(6.3 \pm 3.0) \times 10^{-9}$ & $320 \pm 30$ & & & Richter (1968) \\
\hline Mira $\left(P>250^{\mathrm{d}}\right)$ & $(6.3 \pm 4.7) \times 10^{-9}$ & $640 \pm 140$ & & & Richter (1968) \\
\hline Mira $\left(P>200^{\mathrm{d}}\right)$ & $(2.5 \pm 1.5) \times 10^{-9}$ & $430 \pm 70$ & & & Richter (1968) \\
\hline Mira $\left(P>150^{\mathrm{d}}\right)$ & $(2.5 \pm 0.5) \times 10^{-9}$ & $690 \pm 150$ & & & Richter (1968) \\
\hline Mira $\left(P>90^{\mathrm{d}}\right)$ & & $450 \pm 100$ & & & Richter (1968) \\
\hline Plan. Neb. & $(0.05) \times 10^{-9}$ & $120 \pm 20$ & & & Cahn and Wyatt (1978) \\
\hline
\end{tabular}

Comments: $\rho_{0}, z_{0}$ are derived by assuming a density law of the form (5a), $\rho_{0}^{\prime}, z_{0}^{\prime}$ are derived by assuming a density law of the form $(5 b)$.

the true space densities. The decreasing $z_{0}$-values for systems of longer period can be explained either by different mean ages of the considered groups, or, more likely, as an indication of a preferential orientation parallel to the galactic plane.

It would certainly be rewarding to carry out a more detailed study, which should also take into account the evolutionary status of the EA systems (e.g., Budding's (1983) EA1/EA2, Kholopov's (1981) DM/DS, or Svechnikov et al.'s (1980) RGP/PR/RS groups).

The GCVS becomes a completely insufficient source list when systems with periods of the order of months or years are evaluated: the probability of eclipses is low, and the true space density is much higher than the observed one. A study based on the Catalogue of Spectroscopic Orbits (Batten et al., 1978) was carried out. It comprised systems with $200^{\mathrm{d}} \leqq P \leqq 1000^{\mathrm{d}}$ and evolved (giant) primaries. It was noted that the incompleteness of data for systems with distances $>80 \mathrm{pc}$ makes it impossible to derive the $z$-distribution. The observed $\rho_{0}$ of these systems in the solar neighbourhood is of the order $2.5 \times 10^{-5} \mathrm{pc}^{-3}$. Because of effects of inclination, the true density is somewhat larger. 
The space density of this special group of binaries is of the same order of magnitude as that of the EW systems, only the probability of eclipses is very much reduced. This should be kept in mind when the estimate of Shapley (1948) concerning the frequency of EW systems is quoted. The argument that only EWs and CBs have similar, high space densities (Kraft, 1965) is not valid; the space density of EA systems with giant primaries and periods between 200 and 1000 days is several times higher than that of the CBs.

\subsection{OTHER TYPES OF STARS}

No new studies of the galactic distribution and space density of other types of objects were carried out. Data for Mira variables were taken from Richter (1968); data for late type giants and supergiants were taken from Mikami and Ishida (1981). Results are shown in Table IX.

\section{Discussion of the Results}

\subsection{Statistical tests For eVolutionary DePENDENCES}

When a group of stars evolves from state $i$ into state $k$, the following relations hold for a Galaxy with constant rates of star formation:

$$
\begin{aligned}
& z_{0, i} \leqq z_{0, k} \\
& t_{i} / \Sigma_{i}=t_{k} / \Sigma_{k}
\end{aligned}
$$

where $t_{i}$ is the lifetime in state $i$ and $\Sigma_{i}$ the surface density of objects of type $i$, as defined in (10).

\subsection{Relations BETWEen NOVAE, DWARF NOVAE, AND SUPERNOVAE OF TYPE I}

Let us first examine the possible relationships between different groups of $\mathrm{CBs}$ and their connection with SNI.

Novae and dwarf novae have similar $z$-distributions, the space density of $\mathrm{N}$ is about $\frac{1}{10}$ that of DN. The galactic distribution of these objects indicates that, at least in the solar neighbourhood, the majority of these systems belongs to the older Population I.

In addition to the majority of young objects, a few objects of old age seem to exist. Detached evolved binary systems like V471 Tau and AA Dor (LB 3459) might represent potential CBs that will enter their active state only after billions of years when gravitational radiation or evolutionary expansion of the secondary will transform the systems into semi-detached ones.

Let us review the observational evidence for a nonvanishing fraction of Population II CBs (see also Webbink, 1980):

(a) Nova T Sco in the globular cluster M80 (NGC 6093). It is a fast nova $\left(t_{3}=22^{\mathrm{d}}\right)$ of light curve type A (smooth), and has an absolute magnitude in good agreement with that of other novae of type A, when it is placed at the cluster distance (Sawyer, 1938; Duerbeck, 1981). 
(b) Nova Oph (1938) in the globular cluster M14 (NGC 6402). Its light curve is poorly covered, but it is possibly a slow nova. With an observed maximum brightness of $16^{m}$, it could have been as bright as $10^{m}$ at true maximum, corresponding to $M=-6^{m}$. Membership is likely (Sawyer-Hogg and Wehlau, 1964).

(c) V1148 Sgr near the globular cluster NGC 6553. It is not close to the cluster, it is poorly observed, and membership is unlikely (Mayall, 1949).

Several candidates for dwarf novae in globular clusters are listed by Webbink (1980). A recently found object is V101 in the globular cluster M5 (NGC 5904) (Margon et al., 1981). Assuming cluster membership, their absolute magnitudes are, in general, unusually bright $\left(M^{\max }=+2^{m} .7\right.$ for V101), and it is not unlikely that they are foreground objects. Deeper searches, which should record stars of at least $m=19^{m}$ are necessary to look successfully for DN candidates at maximum light in those globular clusters which are relatively close to the Sun.

A similar dichotomy between young and old members of the class exists in the case of planetary nebulae: formerly assigned to Population II (Oort, 1958), they show a strong concentration towards the galactic plane, with $z_{0}=120 \mathrm{pc}$ (Cahn and Wyatt, 1978), and a much rarer occurrence in Population II aggregates, e.g., the object Küstner 648 in the globular cluster M15 (NGC 7078).

Concerning the scheme $\mathrm{CB} \rightarrow \mathrm{SNI}$, we note that

$$
z_{0, \mathrm{DN}}<z_{0, \mathrm{SNI}} \text {, }
$$

using the $z$-distribution of galactic SNI, as determined by Johnson and MacLeod (1963). Most DN are Population I objects, thus most SN I will also be Population I objects. The existence of SNI in elliptical galaxies can be explained by the fact that $\mathrm{N}$ and probably also DN occur in Population II stellar aggregates.

While relation (15) is thus fulfilled, (16) cannot be checked because of lack of data. However, some conclusions can be drawn from the death rate of cataclysmic binaries.

An estimate of the lifetime of a $\mathrm{CB}$ can be derived from the following arguments: dwarf novae show mean mass transfer rates of $\sim 1.5 \times 10^{-9} M_{\odot} \mathrm{yr}^{-1}$ (Smak, 1982), and mean secondary masses of $\sim 0.5 M_{\odot}$. Assuming that all DN start with $M_{\text {sec }}=1 M_{\odot}$, and that they use up the total mass of the secondary during the DN state, the maximum length of the active state is $6 \times 10^{8} \mathrm{yr}$. It can, however, not be excluded that DN switch, during the course of their evolution, to the nova state and vice versa (Duerbeck, 1980). Novae have mean mass transfer rates that are higher by a factor 10, leading under the above assumptions to a lifetime of $6 \times 10^{7} \mathrm{yr}$. If both types of variability occur in identical systems, it can be concluded from the observed space densities that half of the mass is transferred in the low (DN) state, half during the high (N) state.

The lifetimes of CBs are thus at most between $6 \times 10^{7}$ and $6 \times 10^{8} \mathrm{yr}$. For the following discussion, a mean lifetime of $2 \times 10^{8} \mathrm{yr}$ is adopted.

The total number of DN in the Galaxy can be calculated by Parenago's formula (Richter, 1968). Using the space density $\rho_{0}$ and the scale-height $z_{0}$ as determined in (11) and the radial density gradient $\partial \log \rho / \partial R=-0.24$ (Richter, 1968), Parenago's formula 
yields $1.1 \times 10^{6} \mathrm{DN}$. With a lifetime of $2 \times 10^{8} \mathrm{yr}$, the death rate, one DN in $180 \mathrm{yr}$, is not very far from the estimate of the SN I frequency in the Galaxy.

\subsection{CATAClysmic BINARIES AS DESCENDANTS OF UNEVOLVED BINARIES}

So far, it is not possible to prove, by statistical arguments, evolutionary schemes including normal binaries and CBs. We will, however, collect the statistical evidence.

Case 1: $\mathrm{EW} \rightarrow \mathrm{CB}$

$z_{0, \mathrm{EW}}<z_{0, \mathrm{CB}}$ is valid; in addition, an old population of EWs seems to exist, corresponding to Population II novae (see Sections 4, 6, 9.1, and 10.2).

For the application of Equation (16), estimates of the surface densities and lifetimes must be available. With novae $\frac{1}{10}$ as frequent as dwarf novae, the surface densities are:

$$
\Sigma_{\mathrm{EW}}=49 \times 10^{-4} \mathrm{pc}^{-2} ; \quad \Sigma_{\mathrm{CB}}=2.5 \times 10^{-4} \mathrm{pc}^{-2} .
$$

With $t_{\mathrm{CB}}=2 \times 10^{8} \mathrm{yr}$, the lifetime in the EW state is

$$
t_{\mathrm{EW}}=3.3 \times 10^{9} \mathrm{yr} .
$$

The strong concentration of $\mathrm{EW}$ systems towards the galactic plane suggests a slightly lower value for the mean age of the (young) W UMa population, about $10^{9} \mathrm{yr}$ (Scheffler, 1983). In view of the uncertainties in the derivation, the agreement is surprisingly good, and the result is also not in conflict with most theoretical predictions (see, e.g., Eggleton et al., 1976). It seems worthwhile to further revive and substantiate the scheme $\mathrm{EW} \rightarrow \mathrm{CB}$.

Concerning the older W UMa generation and the occurrence of novae in globular clusters, the following should also be considered:

EW binaries are tightly bound and should survive in globular clusters where gravitational interaction between the cluster members is noticeable. These EW systems would be good candidates for progenitors of CBs in globular clusters. Systems with larger separation going through case $\mathrm{B}$ or $\mathrm{C}$ of binary evolution are more likely subject to disruption; if these systems are the progenitors of $\mathrm{CBs}$, the percentage of $\mathrm{CBs}$ in globular clusters should be noticeably smaller than in the field (of similar age).

A careful search for CBs and eclipsing binaries of all types in globular clusters is a necessary prerequisite for the establishment of evolutionary sequences.

\section{Case 2: $\mathrm{EA} \rightarrow \mathrm{CB}$}

$z_{0, \mathrm{EA}}<z_{0, \mathrm{CB}}$ is valid, but because of a possible preferential orientation of orbital planes, this relation is not conclusive (see Section 9.2).

Equation (16) is difficult to apply because of the unknown value of $z_{0}$ in the case of spectroscopic binaries or the uncertain value of $\rho_{0}$ in the case of eclipsing binaries. Assuming $z_{0, \mathrm{CB}}=z_{0 \text {, EA }}$, the surface densities can be replaced by the space densities, and we obtain, using the space density of spectroscopic binaries:

$$
t_{\mathrm{EA}}=2 \times 10^{8} \times 2.5 \times 10^{-5} / 1.2 \times 10^{-6} .
$$


If we introduce a factor $f$, the fraction of Algol systems which evolve into CBs is

$$
t_{\mathrm{EA}}=f \times 4 \times 10^{9} \mathrm{yr} .
$$

It is assumed that the lifetime of the giant components, for which our statistical study has been carried out, is of the order of $3 \times 10^{7} \mathrm{yr}$ (Meyer-Hofmeister, 1982), the factor $f$ can be determined:

$$
f=7 \times 10^{-3},
$$

i.e., less than $1 \%$ of all Algol systems with giant components and periods between 200 and 1000 days must undergo a spiralling-in in the course of their evolution in order to account for the observed space density of CBs. This very attractive result needs, however, still much more confirmation both from the theoretical and the stellar-statistical side before it can be used as an argument in favour of the scheme EA $\rightarrow$ CB.

\subsection{RECURRENT NOVAE AND SYMBIOTIC STARS}

Finally, a few words on recurrent novae and symbiotic stars are appropriate. These objects seem to have similar $z$-distributions, those of an older disc population. The (not unequivocally accepted) models of both groups, binary systems consisting of a giant and an accreting (white?) dwarf, are also very similar. The red giants in both groups are sometimes long-period variables (RS Oph, RX Pup, V1016 Cyg). The galactic distribution of both groups is similar to that of $M$ giant stars (Mikami and Ishida, 1981) and Mira variables of comparable period (Richter, 1968).

The evolutionary scheme (e.g., Rudak, 1982), that a close, long-period binary having undergone case $\mathrm{C}$ evolution without the formation of a common envelope and thus having avoided a spiralling-in, will become a symbiotic star when the secondary evolves through the giant, or, sometimes, the Mira stage, is in agreement with the galactic distribution.

\section{Conclusion}

From the study of the galactic distribution it is found that novae, dwarf novae, perhaps supernovae of type I

and

recurrent novae and symbiotic stars

form two distinct groups.

Both schemes $\mathrm{EW} \rightarrow \mathrm{CB}$ and $\mathrm{EA} \rightarrow \mathrm{CB}$ are not in disagreement with the galactic distributions of these systems. The importance of observations of these types of stars in globular clusters is stressed. A summary of the newly determined galactic distributions of cataclysmic binaries and related binary and single stars is given in Table IX. 


\section{Acknowledgements}

I thank Messrs F. Th. Lentes and B. Nelles for their untiring computational help, and the colleagues of the Universitätssternwarte München for their interest in an earlier presentation of this topic as a colloquium. The participation at IAU Colloquium No. 80 was made possible by a travel grant of the Deutsche Forschungsgemeinschaft, Bonn-Bad Godesberg (477/832/83(2)).

\section{References}

Allen, C. W.: 1954, Monthly Notices Roy. Astron. Soc, 114, 387.

Allen, C. W.: 1973, Astrophysical Quantities, 3rd edition (and previous editions), Athlone Press, London.

Allen, D. A.: 1979, in F. M. Bateson et al. (eds.), 'Changing Trends in Variable Star Research', IAU Colloq. 46, 125.

Allen, D. A.: 1980, Monthly Notices Roy. Astron. Soc. 192, 521.

Baade, W.: 1958, in D. J. K. O'Connell (ed.), 'Stellar Populations', Ric. Astron. Spec. Vatican 5, 165.

Bailey, J.: 1981, Monthly Notices Roy. Astron. Soc. 197, 31.

Barlow, M. J., Brodie, J. P., Brunt, C. C., Hanes, D. A., Hill, P. W., Mayo, S. K., Pringle, J. E., Ward, M. J., Watson, M. G., Whelan, J. A. J., and Willis, A. J.: 1981, Monthly Notices Roy. Astron. Soc. $195,61$.

Batten, A. H., Fletcher, J. M., and Mann, P. J.: 1978, Publ. Dominion Astrophys. Obs. Victoria $15,121$. Bruch, A.: 1982, Ph.D. thesis, Münster University.

Bruch, A., Duerbeck, H. W., and Seitter, W. C.: 1981, Mitt. Astron. Ges. 52, 34.

Budding, E.: 1983, Astrophys. Space Sci. 99, (this issue).

Cahn, J. H. and Wyatt, S. P.: 1978, in Y. Terzian (ed.), 'Planetary Nebulae', IAU Symp. 76, 3.

Dinerstein, H. and Hoffleit, D.: 1973, Inf. Bull. Var. Stars, No. 845.

Duerbeck, H. W.: 1980, Habilitation thesis, Bonn.

Duerbeck, H. W.: 1981, Publ. Astron. Soc. Pacific 93, 165.

Duerbeck, H. W. and Seitter, W. C.: 1979, ESO Messenger 17, 1; see also Mitt. Astron. Ges. 50, 90 (1980). Duerbeck, H. W. and Seitter, W. C.: 1982, in K. Schaifers and H. H. Voigt (eds.), Landolt-Börnstein N.S. VI/2, 197.

Eggleton, P. P.: 1983, in M. Livio and G. Shaviv (eds.), 'Cataclysmic Variables and Related Objects', $I A U$ Colloq. 72, 239.

Eggleton, P. P., Mitton, S., and Whelan, J. A. J. (eds.): 1976, in 'Structure and Evolution of Close Binary Stars', IAU Symp. 73.

Garrison, R. F., Hiltner, W. A., and Schild, R. E.: 1982, IAU Circ., No. 3730.

Hoffmann, M.: 1983, private communication.

Hudec, R.: 1981, Bull. Astron. Inst. Czech. 32, 93.

Iwanowska, W. and Burnicki, A.: 1962, Bull. Acad. Pol. Sci. Ser. Sci. Math. Astron. Phys. 10 (10), 537. Johnson, H. M. and MacLeod, J. M.: 1963, Publ. Astron. Soc. Pacific 75, 123.

Kamper, K. W.: 1979, in H. M. van Horn and V. Weidemann (eds.), 'White Dwarfs and Variable Degenerate Stars', IAU Colloq. 53, 494.

Kholopov, P. N.: 1981, Perem. Zvezdy 21, 465.

Kopylov, I. M.: 1957, in G. H. Herbig (ed.), 'Non-Stable Stars', IAU Symp. 3, 71.

Kraft, R. P.: 1962, Astrophys. J. 135, 408.

Kraft, R. P.: 1965, Astrophys. J. 142, 1588.

Kraft, R. P.: 1967, Publ. Astron. Soc. Pacific 79, 395.

Kraft, R. P. and Luyten, W.: 1965, Astrophys. J. 142, 1041.

Kukarkin, B. W.: 1954, Untersuchung der Struktur und Entwicklung der Sternsysteme auf der Grundlage des Studiums veränderlicher Sterne, Akademie-Verlag, Berlin.

Law, W. Y. and Ritter, H.: 1983, Astron. Astrophys. 123, 33.

Margon, B., Downes, R. A., and Gunn, J. E.: 1981, Astrophys. J. 247, L89.

Mayall, M. W.: 1949, Publ. Astron. Soc. Pacific 54, 191.

Meyer-Hofmeister, E.: 1982, in K. Schaifers and H. H. Voigt (eds.), Landolt-Börnstein N.S. VI/2b, 152. 
Mikami, T. and Ishida, K.: 1981, Publ. Astron. Soc. Japan 33, 135.

Moffat, A. F.: 1982, private communication.

Neckel, Th.: 1967, Veröff. Landssternwarte Heidelberg 19.

Neckel, Th. and Klare, G.: 1980, Astron. Astrophys. Suppl. 42, 251.

Oort, J. H.: 1958, in D. J. K. O'Connell (ed.), 'Stellar Populations', Ric. Astron. Spec. Vatican. 5, 415.

Paczynski, B.: 1976, in P. Eggleton, S. Mitton, and J. A. J. Whelan (eds.), 'Structure and Evolution of Close Binary Systems', IAU Symp. 73, 27.

Paczynski, B.: 1980, in P. A. Weyman (ed.), Highlights of Astronomy 5, D. Reidel Publ. Co., Dordrecht, Holland, p. 27.

Parenago, P. P.: 1955, in 'Principes fondamentaux de classification stellaire', Colloque International du CNRS, CNRS, Paris, p. 13.

Payne-Gaposchkin, C. H.: 1977, in M. Friedjung (ed.), Novae and Related Stars, D. Reidel Publ. Co., Dordrecht, Holland, p. 3.

Plaut, L.: 1965, in A. Blaauw and M. Schmidt (eds.), Stars and Stellar Systems V, University of Chicago Press, Chicago, p. 311.

Popov, M. V.: 1964, Perem. Zvezdy 15, 115.

Primkulov, Sh.: 1968, Tsirk. Astron. Tashkent 8, No. 255.

Richter, G.: 1968, Veröffentl. Sternw. Sonneberg 7, 229.

Richter, G.: 1983, Inf. Bull. Var. Stars, No. 2267.

Ritter, H.: 1976, Monthly Notices Roy. Astron. Soc. 175, 279.

Ritter, H.: 1983, Catalogue of Cataclysmic Binaries, MPI Astrophysik Garching, (MPA-51).

Robinson, E. L.: 1975, Astron. J. 80, 515.

Rosino, L.: 1973, Astron. Astrophys. Suppl. 9, 347.

Rudak, B.: 1982, in M. Friedjung and R. Viotti (eds.), 'The Nature of Symbiotic Stars', IAU Colloq. 70, 275.

Sahade, J.: 1959, Liège Symp. No. 9, 'Modèles d'étoiles et évolution stellaire', Mém. Soc. R. Liège Sér. 5, t. 3, p. 76 .

Sawyer, H.: 1938, J. Roy. Astron. Soc. Canada 32, 69.

Sawyer-Hogg, H. and Wehlau, A.: 1964, J. Roy. Astron. Soc. Can. 58, 163.

Scheffler, H.: 1983, in K. Schaifers and H. H. Voigt (eds.), Landolt-Börnstein N.S. VI/2c, 175.

Schmidt-Kaler, Th.: 1957, Z. Astrophys. 41, 182.

Schmidt-Kaler, Th.: 1962, Kl. Veröffentl. Remeis-Sternw. Bamberg 34. 109.

Schmidt-Kaler, Th.: 1982, in K. Schaifers and H. H. Voigt (eds.), Landolt-Börnstein N. S. VI/2b, 1.

Shapley, H.: 1948, Harvard Obs. Monograph 7, 249.

Shu, F. H.: 1980, in M. J. Plavec, D. M. Popper, and R. K. Ulrich (eds.), 'Close Binary Stars: Observations and Interpretation', IAU Symp. 88, 477.

Smak, J.: 1982, Acta Astron. 32, 213.

Starrfield, S., Truran, J. W., and Sparks, W. M.: 1981, Astrophys. J. 243, L27.

Svetchnikov, M. A., Istomin, L. F., and Grehova, O. A.: 1980, Perem. Zvezdy 21, 399.

Trimble, V.: 1983, Nature 303, 137.

Tsvetkov, D. Yu.: 1981, Soviet Astron. Letters 7, 254.

Upgren, A. R.: 1963, Astron. J. 68, 475.

Van 't Veer, F.: 1975, Astron. Astrophys. 40, 167.

Vasilevskis, S., Harlan, E. A., Klemola, A. R., and Wirtanen, C. A.: 1975, Publ. Lick Obs. 22, Pt. 5.

Vidal, N. V. and Rodgers, A. W.: 1974, Publ. Astron. Soc. Pacific 86, 26.

Vilhu, O.: 1981, Astrophys. Space Sci. 78, 401.

Voikhanskaya, N. F.: 1973, Astrofiz. Issled 5, 89.

Vogt, N.: 1981, Habilitation thesis, Bochum University.

Wallerstein, G.: 1981, Observatory 101, 172.

Warner, B.: 1974a, Monthly Notices Roy. Astron. Soc. 167, 61p.

Warner, B.: 1974b, Monthly Notices Astron. Soc. Southern Africa 33, 21.

Webbink, R. F.: 1976, Astrophys. J. 209, 829.

Webbink, R. F.: 1978, Publ. Astron. Soc. Pacific 90, 57.

Webbink, R. F.: 1980, in M. J. Plavec, D. M. Popper, and R. K. Ulrich (eds.), 'Close Binary Stars: Observations and Interpretation', IAU Symp. 88, 561.

Wenzel, W. and Meinunger, I.: 1978, Astron. Nachr. 299, 237.

Yungelson, L. R.: 1982, in J. Tremko (ed.), Ejection and Accretion of Matter in Binary Systems, Veda, Bratislava, p. 11. 